\title{
Grave's Eye Ophthalmopathy Developing Following Radioiodine Therapy for Toxic Adenoma
}

\author{
Hala Chafic Ahmadieh ${ }^{1 *}$, Ibrahim Salti ${ }^{2 *}$ \\ ${ }^{1}$ American University of Beirut-Medical Center, Riad El-Solh, Beirut, Lebanon \\ ${ }^{2}$ Department of Internal Medicine, Division of Endocrinology and Metabolism, \\ American University of Beirut-Medical Center, Beirut, Lebanon \\ Email: ha76@aub.edu.lb, isalti@aub.edu.lb
}

Received July 6, 2013; revised August 29, 2013; accepted September 10, 2013

Copyright (c) 2013 Hala Chafic Ahmadieh, Ibrahim Salti. This is an open access article distributed under the Creative Commons Attribution License, which permits unrestricted use, distribution, and reproduction in any medium, provided the original work is properly cited.

\begin{abstract}
The development of Grave's ophthalmopathy following radioiodine treatment for Grave's disease is well recognized. However, development of Grave's ophthalmopathy following radioiodine therapy given for toxic adenoma or toxic nodular goiter has been reported but very rarely. Hereby, we reported a case of a 69-year-old male patient who developed ophthalmopathy after receiving $30 \mathrm{mCi}(1110 \mathrm{mBq}){ }^{131}$ I radioiodine treatment for a toxic nodule. In addition, a review of the current literature with regards to this topic is presented.
\end{abstract}

Keywords: Grave’s Ophthalmopathy; Toxic Adenoma; Toxic Nodular Goiter; Radioiodine Therapy

\section{Introduction}

The development of Grave's ophthalmopathy following radioiodine treatment for Grave's disease, although controversial, is well recognized, where in a systematic review RAI for Graves' disease was associated with increased risk of occurrence or progression of ophthalmopathy when compared with ATD or surgery. It may impair the quality of life [1-9]. The clinical findings of ophthalmopathy may be unilateral, bilateral, symmetric, or asymmetric. Occasionally, a unilateral ophthalmopathy precedes bilateral involvement. Clinical manifestations usually vary from patient to patient, and even in the same patient over time and they include proptosis, swelling of the periorbital tissues, redness of the eyes, impairment of eye movement, abrasions, and optic nerve damage. Accompanying eye symptoms usually include excessive eye tearing and sensitivity to light; increased burning, gritty, or pulling sensations; double vision, pain because of abrasion, and it may even lead to loss of visual acuity. In Graves’ ophthalmopathy, the extraocular muscles become enlarged, firm and rubbery and the muscle volume may be increased 8 to 10 times the normal size. This increased muscle volume causes forward displacement of the eyeball, resulting in proptosis [10]. Development of opthalmopathy post radioiodine treatment for toxic or non-toxic

"Both authors contributed in the writing process. multinodular goiter has been very rarely reported previously. We report a case of a 69-year-old male patient who developed ophthalmopathy after receiving $30 \mathrm{mCi}$ (1110 $\mathrm{mBq}$ ) of ${ }^{131} \mathrm{I}$ radioiodine treatment for a toxic nodule which was causing overt hyperthyroidism. A review of the previously reported cases and the postulated mechanisms explaining the development of ophthalmopathy in such patients is provided.

\section{Case Report}

A 69-year-old male patient was known to have hyperthyroidism diagnosed 10 years ago in a different institution and to which he received methimazole therapy for 1 year, after which he was clinically and biochemically euthyroid for a total of 9 years, presented in 2012 with a one month history of palpitations, weight loss, heat intolerance and decreased appetite. Thyroid function tests confirmed thyrotoxicosis (see Table 1). The patient had a past medical history of hypertension, dyslipidemia, atrial fibrillation diagnosed 6 years ago, with one failed cardio-version attempt, which was followed by electro-frequency ablation therapy 2 years previously and he was maintained on warfarin, gemfibrozil, pantoprazole and sotalol. He was a non-smoker. Family history was positive for hypothyroidism in his sister.

On physical exam he was found to have a resting fine 
Table 1. Summary of thyroid function tests since presentation and following radioiodine therapy.

\begin{tabular}{|c|c|c|c|c|c|}
\hline & Normal values & Upon presentation & $\begin{array}{l}1 \text { week after } \\
\text { methimazole }\end{array}$ & $\begin{array}{l}2 \text { months after } \\
\text { radiotherapy }\end{array}$ & $\begin{array}{c}5 \text { months after } \\
\text { radiotherapy }\end{array}$ \\
\hline TSH (uU/mL) & $0.27-4.2$ & 0.007 & 0.008 & 7.32 & 78 \\
\hline Free T4 (ng/dl) & $0.93-1.7$ & 1.72 & 1.60 & 0.46 & 0.81 \\
\hline Free T3 (pg/ml) & $1.8-4.6$ & 4.06 & 3.7 & 1.58 & 1.2 \\
\hline Anti-TPO Antibody(IU/ml) & $<30$ & 359.1 & & $>1000$ & \\
\hline TRAbs (U/L) & (>1.5, positive) & & & 5.88 & 5.73 \\
\hline ESR & & 4 & & & \\
\hline
\end{tabular}

tremor, tachycardia with a baseline regular heart rate of 110 beats per minute. There was no evidence of thyroid eye disease then. Neck palpation revealed a single right thyroid nodule. Ultrasound thyroid revealed enlarged right thyroid harboring one nodule $(2 \times 0.6 \mathrm{~cm})$ (see Figure 1). Thyroid scan showed increased uptake in the right lobe suggestive of a toxic adenoma (see Figure 2). He was started on methimazole $15 \mathrm{mg}$ daily for a total of 1 week after which methimazole was stopped for 3 days and then the patient received $30 \mathrm{mCi}$ of radioiodine therapy. One week later patient noted the appearance of conjunctivalredness together with diplopia on vertical and horizontal gaze and blurred vision. MRI of the orbit revealed a 7 $\mathrm{mm}$ proptosis and extra-ocular muscle hypertrophy (see Figure 3). Two months later, the patient developed signs and symptoms of hypothyroidism after which he was started on LT4 therapy. Patient was then started on oral prednisone $50 \mathrm{mg}$ daily but patient's symptoms did not improve. He was then hospitalized for high dose pulse glucocorticoid therapy consisting of 1 gram of intravenous methyl-prednisolone daily for 4 days, after which he started noting minor improvement in his eye redness and swelling.

\section{Discussion}

Radioiodine therapy has been widely used for the treatment of Graves' disease for several decades, but the association between radioiodine therapy and development or worsening of Graves' ophthalmopathy is still not very well understood. Some retrospective studies did report an association but others did not [1-8]. A systematic review was conducted and showed that RAI for Graves' disease was associated with increased risk of occurrence or progression of ophthalmopathy when compared with ATD or surgery. The risk of Graves' ophthalmopathy was around $20 \%$ after radioiodine and 5\% after anti-thyroid drugs. The risk of developing severe ophthalmopathy in Graves' disease patients after radioiodine therapy was around $7 \%$ [9].
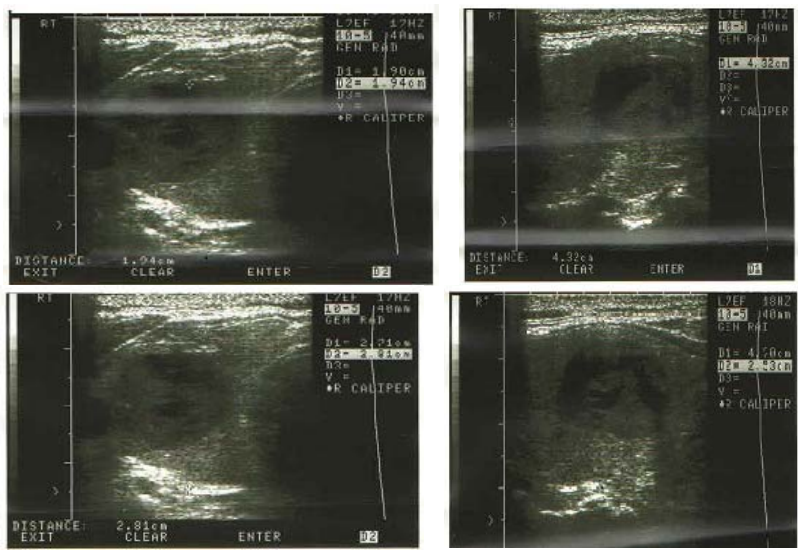

Figure 1. Ultrasound of thyroid of the patient.

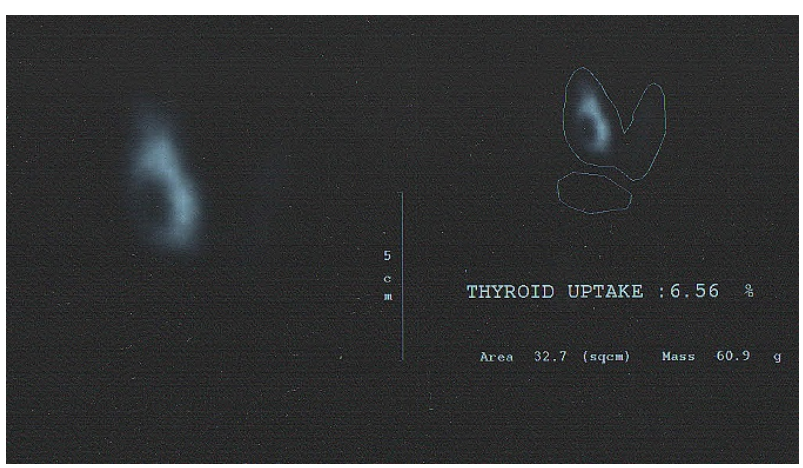

Figure 2. Thyroid scan of the patient.

The development of Graves' ophthalmopathy post radioiodine treatment for multi-nodular goiter (toxic or nontoxic) has been rarely described [11-13]. Presence of TRAb has been described in $15 \%$ - 52\% of patients with toxic and non-toxic nodular goiter [3-16]. It is possible that such patients may be harboring sub-clinical Grave's disease which may be uncovered after radio-iodine therapy. Wallaschofski et al. noted that TRAbs was detected in $36 \%$ (4 of 11) of patients with TMG type A, defined as diffuse asymmetrical multinodular goiter, after RAI therapy, despite the fact that those patients had negative 

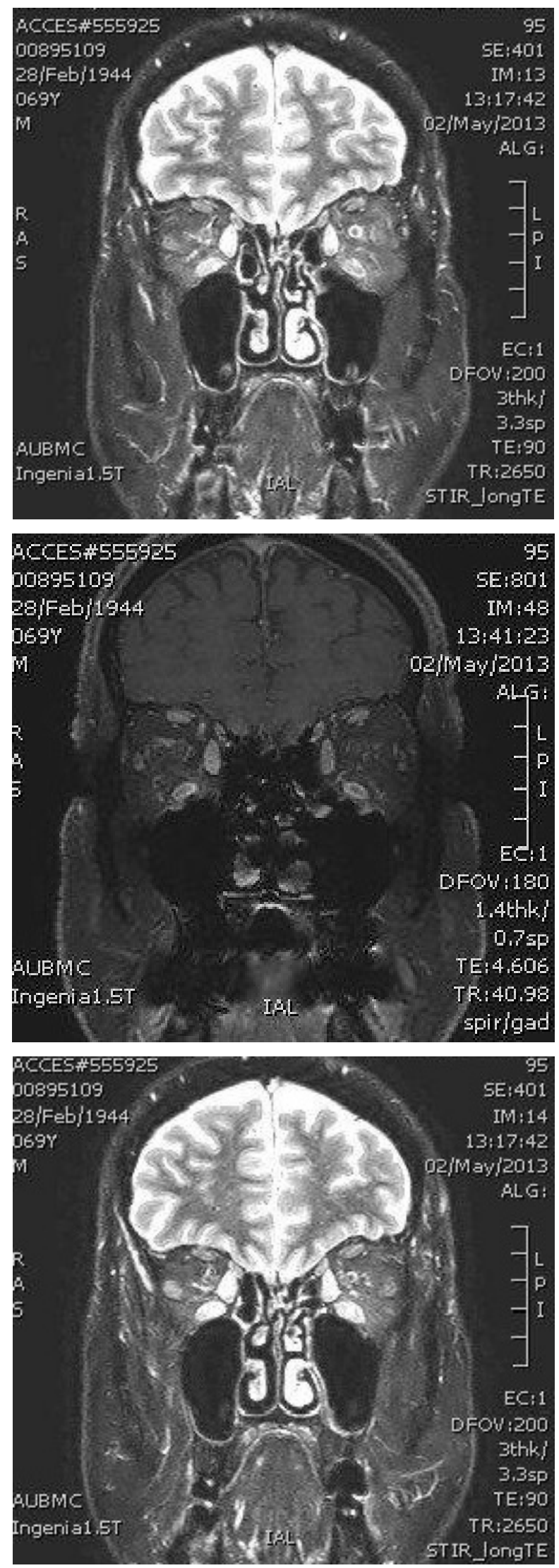

Figure 3. MRI of orbit of the patient.
TRAbs prior to the radioiodine. On the other hand TRAbs were not detectable in patients with Toxic adenoma and TMG Type B, defined as discrete multiple nodules in the thyroid gland. Furthermore this study showed that 3 out of those 4 patients, who had detectable TRAbs after radioiodine, had positive anti-TPO-Abs before RAI therapy was given. These findings provide further evidence for pre-existing grave's disease in patients with toxic multi-nodular goiter especially those who had high anti-TPO-Abs at baseline, and seemed to be at increased risk of developing TRAbs or thyroid associated ophthalmopathy post radioiodine therapy [16]. Orsolon et al. also described the development of TRAbs and the increased in TRAbs titers, in those who had baseline TRAb, following radioiodine therapy for either toxic or non-toxic multi nodular goiter [17]. Van Leussen reported a case similar to our patient, who developed severe Graves' ophthalmopathy, post radioiodine therapy, given for toxic multinodular goiter, necessitating treatment with oral steroids and orbital radiotherapy [18].

It was previously postulated that radioiodine therapy could be inducing the release of TSH receptor antigens into the circulation which in turn would trigger the development of TSH receptor antibody (TRAb) [11-17].

This case highlights that severe Graves' opthalmopathy can develop in patients who receive radioiodine therapy for toxic adenoma due to possible concomitant presence of Graves's disease. Our patient developed positive TRAb post radioiodine therapy given for his toxic adenoma. This was contrary to the study presented by Wallaschofski et al. who reported lack of positivity of TRAb in patients with toxic adenoma who developed orbitopathy post radioiodine therapy. However this was similar to the finding presented by Orsolon et al. However our patient did have positive anti-TPO-Abs prior to RAI therapy, confirming the fact that a dormant autoimmune disease was mainly responsible for the subsequent development of Graves’ ophthalmopathy.

\section{Conclusion}

This case highlights that Graves' ophthalmopathy could actually develop post radioiodine treatment for toxic adenoma or toxic multinodular goiter. Therefore endocrinologists, ophthalmologists and general physicians should be aware of this possibility although the incidence is really very low.

\section{REFERENCES}

[1] J. Kriss, V. Pleshakov, A. L. Rosenblum, M. Holderness, G. Sharp and R. Utiger, "Studies on Pathogenesis of the Ophthalmopathy of Graves' Disease,” Journal of Clinical Endocrinology and Metabolism, Vol. 27, No. 4, 1967, pp. 582-593. http://dx.doi.org/10.1210/jcem-27-4-582 
[2] J. Barbosa, E. Wong and R. P. Doe, "Ophthalmopathy of Graves’ Disease: Outcome after Treatment with Radioactive Iodine, Surgery or Antithyroid Drugs," Archives of Internal Medicine, Vol. 130, No. 1, 1972, pp. 111-113. http://dx.doi.org/10.1001/archinte.1972.03650010097018

[3] H. Vestergaard and P. Laurberg, "Radioiodine and Aggravation of Graves' Ophthalmopathy,” Lancet, Vol. 334, No. 8653, 1989, pp. 653-647.

[4] A. Barth, P. Probst and H. Bürgi, "Identification of a Subgroup of Graves' Disease Patients at Higher Risk for Severe Ophthalmopathy after Radioiodine,” Journal of Endocrinological Investigation, Vol. 14, No. 3, 1991, pp. 209212.

[5] R. D. Hamilton, W. E. Mayberry, W. M. McConahey and K. C. Hanson, “Ophthalmopathy of Graves’ Disease; a Comparison between Patients Treated Surgically and Patients treated with Radioiodine,” Mayo Clinic Proceedings, Vol. 42, 1967, pp. 812-818.

[6] B. M. Calissendorff, M. Söderström and A. Alveryd, "Ophthalmopathy and Hyperthyroidism: A Comparison between Patients Receiving Different Antithyroid Treatment,” Acta Ophthalmologica (Copenhagen), Vol. 64, No. 6, 1986, pp. 698-703. http://dx.doi.org/10.1111/j.1755-3768.1986.tb00688.x

[7] V. Sridama and L. J. DeGroot, "Treatment of Graves' Disease and the Course of Ophthalmopathy," American Journal of Medicine, Vol. 87, No. 1, 1989, pp. 70-73. http://dx.doi.org/10.1016/S0002-9343(89)80485-1

[8] H. J. Aranow and R. M. Day, "Management of Thyrotoxicosis in Patients with Ophthalmopathy: Antithyroid Regimen Determined Primarily by Ocular,” Journal of Endocrinology and Metabolism, Vol. 25, 1965, pp. 1-10. http://dx.doi.org/10.1210/jcem-25-1-1

[9] S. Acharya, "Radioiodine Therapy (RAI) for Graves’ Disease (GD) and the Effect on Ophthalmopathy: A Systematic Review,” Clinical Endocrinology, Vol. 69, No. 6, 2008, pp. 943-950.

[10] R. Bahn, “Graves' Ophthalmopathy,” The New England Journal of Medicine, Vol. 362, 2010, pp. 726-738. http://dx.doi.org/10.1056/NEJMra0905750

[11] L. Chiovato, F. Santini, P. Vitti, C. Bendinelli and A. Pinchera, “Appearance of Thyroid Stimulating Antibody and
Graves' Disease after Radioiodine Therapy for Toxic Nodular Goiter,” Clinical Endocrinology (Oxf), Vol. 40, No. 6, 1994, pp. 803-806. http://dx.doi.org/10.1111/j.1365-2265.1994.tb02516.x

[12] B. Nygaard, R. A. Metcalfe, J. Phipps, A. P. Weetman and L. Hegedüs, “Graves' Disease and Thyroid Associated Ophthalmopathy Triggered by 131I Treatment of Non-Toxic Goiter," Journal of Endocrinological Investigation, Vol. 22, No. 6, 1999, pp. 481-485.

[13] A. A. Tahrani, S. Rangan and P. Moulik, "Grave’s Eye Disease Developing Following Radioiodine Treatment for Toxic Nodular Goiter,” Experimental and Clinical Endocrinology \& Diabetes, Vol. 115, No. 7, 2007, pp. 471-473. http://dx.doi.org/10.1055/s-2007-970400

[14] I. B. Pedersen, N. Knudsen, H. Perrild, L. Ovesen and P. Laurberg, "TSH-Receptor Antibody Measurement for Differentiation of Hyperthyroidism into Graves' Disease and Multinodular Toxic Goitre: A Comparison of Two Competitive Binding Assays,” Clinical Endocrinology (Oxf), Vol. 55, No. 3, 2001, pp. 381-390.

http://dx.doi.org/10.1046/j.1365-2265.2001.01347.x

[15] P. P. Smyth, D. Neylan and D. K. O’Donovan, “The Prevalence of Thyroid-Stimulating Antibodies in Goitrous Disease Assessed by Cytochemical Section Bioassay," The Journal of Clinical Endocrinology \& Metabolism, Vol. 54, No. 2, 1982, pp. 357-361. http://dx.doi.org/10.1210/jcem-54-2-357

[16] H. Wallaschofski, D. Müller, P. Georgi and R. Paschke, "Induction of TSH-Receptor Antibodies in Patients with Toxic Multinodulargoitre by Radioiodine Treatment," Hormone and Metabolic Research, Vol. 34, No. 1, 2002, pp. 36-39. http://dx.doi.org/10.1055/s-2002-19965

[17] P. Orsolon, A. Lupi, G. De Antoni Migliorati and A. Vianello Dri, “Appearance of Graves'-Like Disease Following Regression of Autonomously Functioning Thyroid Nodules. Two Case Reports,” Minerva Endocrinology, Vol. 23, No. 2, 1998, pp. 53-56.

[18] J. J. van Leussen, M. A. Edelbroek, M. A. Talsma and L. J. de Heide, “Graves’ Disease Induced by $\mathrm{Na}(131) \mathrm{I}$ Therapy for Toxic Multinodulargoiter,” The Netherlands Journal of Medicine, Vol. 57, No. 5, 2007, pp. 194-197. http://dx.doi.org/10.1016/S0300-2977(00)00068-1

\section{Abbreviations Used:}

mCi: Millicurie

$\mathrm{mBq}$ : Megabecquerel

MRI: Magnetic Resonance Imaging

LT4: L-Thyroxine

TSH: Thyroid Stimulating Hormone

TRab: Anti Thyroid Hormone Receptor Antibody
Anti TPO: Anti Thyroid Peroxidase Antibody

Anti TG: Anti Thyroglobulin

TMG: Toxic Multi Nodular Goiter

RAI: Radioiodine 